\title{
Author Correction: A recellularized human colon model identifies cancer driver genes
}

Huanhuan Joyce Chen, Zhubo Wei, Jian Sun, Asmita Bhattacharya, David J Savage, Rita Serda, Yuri Mackeyev, Steven A Curley, Pengcheng Bu, Lihua Wang, Shuibing Chen, Leona Cohen-Gould, Emina Huang, Xiling Shen,

Steven M Lipkin, Neal G Copeland, Nancy A Jenkins and Michael L Shuler

Correction to: Nature Biotechnology https://doi.org/10.1038/nbt.3586, published online 11 July 2016.

In the version of this article initially published, NCI (NIH) grant R01 CA157663 to E.H. was missing from the Acknowledgements. The error has not been corrected in the original article.

Published online: 3 June 2019

https://doi.org/10.1038/s41587-019-0163-6

\section{Author Correction: Circularly permuted and PAM-modified Cas9 variants broaden the targeting scope of base editors}

Tony P. Huang, Kevin T. Zhao (D), Shannon M. Miller, Nicole M. Gaudelli, Benjamin L. Oakes, Christof Fellmann (D), David F. Savage and David R. Liu (D)

Correction to: Nature Biotechnology https://doi.org/10.1038/s41587-019-0134-y, published online 20 May 2019.

In the version of this article initially published, another technique for CBEs should have been included in the sentence "The activity window of base editors in mammalian cells has proven surprisingly difficult to broaden, with multimeric deaminase assembly for CBEs ${ }^{33}$ and extended guide RNAs for $\mathrm{ABEs}^{34}$ representing the only window-broadening strategies reported to date." Linker variation ${ }^{31}$ has been added. The error has been corrected in the PDF and HTML versions of this article.

Published online: 10 June 2019

https://doi.org/10.1038/s41587-019-0168-1

\section{Publisher Correction: Biotech's baby boom}

John Hodgson

Correction to: Nature Biotechnology https://doi.org/10.1038/s41587-019-0112-4, published online 3 May 2019.

In the version of this article initially published, Greg Bailey was described as the CEO of Juvenescence AI. He is the CEO of Juvenescence. The error has been corrected in the HTML and PDF versions of the article. 